\title{
Architecture Singularities in Flagged Parallel Manipulators
}

\author{
Júlia Borràs, Federico Thomas, and Carme Torras
}

\begin{abstract}
Flagged manipulators are of interest because they are the only Stewart-Gough platforms for which a cell decomposition of their singularity loci is available. Here we show that the known family of such manipulators can be enlarged if one allows robot designs that, for some particular parameter values, become architecturally singular. Along this line, the most general 6-6 flagged manipulator is derived by applying a singularity-preserving transformation that leaves the relative position between two lines invariant. This transformation opens up the possibility of an "equal cross ratios" architectural singularity, which is shown to appear clearly in the factorization of the jacobian determinant. From the 6-6 flagged manipulator, all the extended family of (possibly architecturally-singular) flagged manipulators is derived.
\end{abstract}

Index Terms-Parallel manipulators, robot kinematics, architecture singularities, kinematics singularities, manipulator design.

\section{INTRODUCTION}

A great deal of research has been performed on the singularity analysis of the Stewart-Gough platform and its numerous specializations, but it was not until recently that the analytic form of the six-dimensional singularity locus of the general platform has been obtained [9]. This provides an analytic characterization of singularities, but it does not say much about their nature and the topology of their singularity loci in the configuration space of the platform with respect to the base.

While the nature of singularities for some specializations (designs in which the endpoints of the legs merge into multiple spherical pairs) has been successfully addressed by using Grassmann line geometry [11], [15], their topological study remains largely as an important open problem [4].

Only in the case of flagged manipulators, a cell decomposition of their singularity loci has been derived [14], [1]. This complete characterization of the arrangement of singularity hypersurfaces in the configuration space permits identifying the different nonsingular regions separated by singularities, the maneuverability restriction occurring in each singular region, as well as the adjacencies between all nonsingular and singular regions. This is useful for manipulator design, including the use of redundant actuators to eliminate certain singularities [2], and also to plan trajectories away from singularities.

Flagged parallel manipulators are characterized by the relation between two flags adequately placed on their platform

This research has been partially supported by the Spanish Committee for Science and Technology (CICYT), project DPI2007-60858, and the Catalan Research Commission, through the Consolidated Robotics Group.

The authors are with the Institut de Robòtica i Informàtica Industrial (CSIC-UPC), Llorens Artigas 4-6, 08028 Barcelona, Spain. \{jborras, torras, fthomas\}eiri.upc.edu

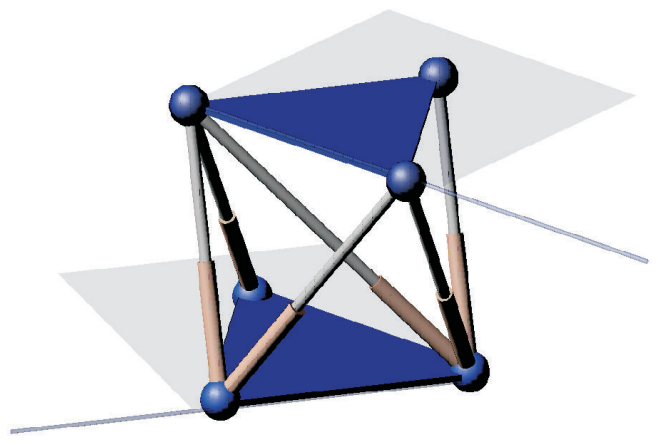

Fig. 1. The basic 3-3 flagged manipulator with the attached flags.

and base, a flag being a triple (point, line, plane) with the point contained in the line which in turn lies on the plane. Figure 1 shows the 3-3 basic flagged manipulator and its attached flags. The key point about such manipulators is that their singularity loci inherit a well-behaved structure from the stratification of the flag manifold [14], which can be described in terms of incidences between the elements (point, line and plane) of the two flags.

With the aim of enlarging the set of manipulators sharing the well-behaved topology of singularities characteristic of flagged manipulators, in this paper we analyze manipulator designs that, for some parameter values, become architecturally singular. These same designs, without such conjunction of values, are flagged manipulators. Thus, by opening up the possibility of architecturally singular instances, the previously derived family of flagged manipulators [1] is considerably enlarged.

Architecture singularities of parallel manipulators were introduced by Ma and Angeles [10]. Later, Husty and Karger [6] showed that there are nine types of such singularities for the general spatial Stewart-Gough platforms. In the special case of manipulators with planar base and platform, there are additional architecture singularities if a projectivity relating both planes satisfies some conditions [7]. Examples are manipulators whose platform and base are affinely equivalent, similar or even congruent, such as the Griffis-Duffy type platform [5].

It is worth noting that manipulators having four points aligned either in the platform or in the base have only the architecture singularities of spatial manipulators and not the additional singularities just mentioned, even if they have planar platform and base [7]. This is the case of flagged manipulators. Therefore, we will only need to consider the 
nine types of architecture singularities mentioned above [6]. Furthermore, only two such types depend on metric conditions (as opposed to leg endpoind junctions and alignments), thus opening up the possibility of architecturally non-singular designs, which are those of interest to us. One such type requires the existence of a one-to-one mapping between 5 aligned endpoints in the platform and the conic section uniquely defined by their corresponding endpoints lying in general position in the base plane [6]. Since this last condition cannot be satisfied by flagged manipulators, we are left with only the other type, namely the "equal cross ratios" singularity.

Then, a transformation of parallel robot designs preserving their singularity loci, but introducing the "equal cross ratios" singularity, permits deriving the (possibly architecturallysingular) 6-6 flagged manipulator, from which other flagged designs are afterwards obtained. In this way, we have enlarged the family of robot designs for which a cell decomposition of their singularity loci is available.

The paper is structured as follows. Section II describes the above-mentioned kinematic transformation and its application to the 3-3 flagged design to come up with the most general 6-6 flagged manipulator. The following section studies the forward kinematics and the singularities of this 6-6 manipulator. Then, in Section IV, the whole family of flagged manipulators consisting of 39 instances is derived. Finally, some conclusions and prospects for future research are drawn in Section V.

\section{FROM THE BASIC 3-3 FLAGGED DESIGN TO THE GENERAL 6-6 FLAGGED MANIPULATOR}

A parallel manipulator is flagged if, and only if, the determinant of its jacobian can be factorized into three terms whose geometric interpretation corresponds to incidences of the kind point-plane, line-line, and plane-point in such a way that all involved points, lines and planes form two flags attached to the base and the platform (Figure 1).

In this section we will derive a 6-6 manipulator design from the basic 3-3 flagged manipulator, and in the next section we will show that it is indeed flagged.

\section{A. Decomposing the basic flagged parallel manipulator into components}

To simplify the kinematic analysis of a Stewart-Gough platform, we can always try to decompose it into independent rigid components. This decomposition is of particular interest when the joints, either on the base or the platform, merge into multiple spherical joints [8]. For a component to be rigid, its number of d.o.f. must equal the number of its actuators. Note that the degrees of freedom of motion of a point relative to a point, a line and a body are 1, 2, and 3, respectively; and the degrees of freedom of a line relative to a line and a body are 4, and 5, respectively. Then, the following five classes of components can be considered:

$P P$ : Point-point (a single leg);

$P L$ : Point-line (two legs sharing a spherical joint);

$P B$ : Point-body (three legs sharing a spherical joint);
$L L$ : Line-line (four legs, their endpoints lying on two lines); and

$L B$ : Line-body (five legs, an endpoint from each lying on a line).

A classification of all possible manipulators listed in terms of combinations of the above components can be found in [8].

The basic flagged manipulator can be seen as the combination of a component of type $L L$, and two components of type PP (Figure 2). Next, we will show how it is possible to move the joints along the two lines involved in the $L L$ component without modifying the kinematic properties of the 3-3 basic flagged manipulator. This transformation will allow us to pass from the basic 3-3 flagged manipulator to a 6-6 flagged design.

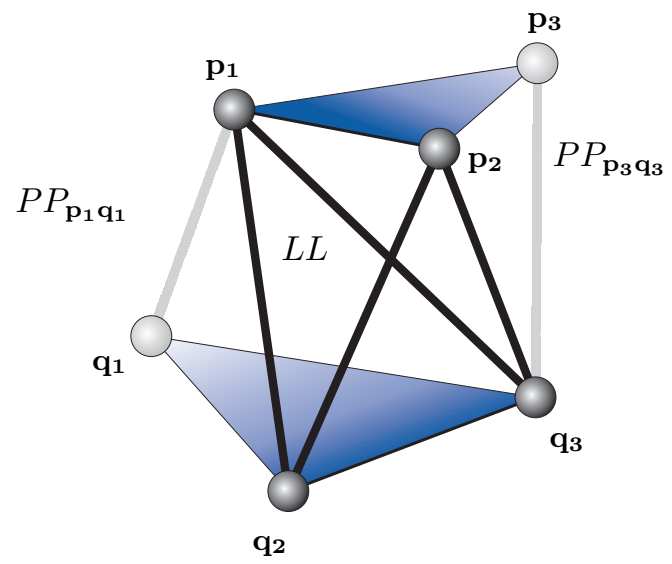

Fig. 2. The basic flagged manipulator can be seen as the combination of a component of type $L L$, and two components of type $P P$.

\section{B. The LL-transformation}

In kinematic terms, a given line in three-dimensional Euclidean space has four degrees of freedom of motion with respect to any other arbitrary line. In other words, by fixing the distances between four couples of points on two lines, as shown in Figure 3, both lines remain rigidly linked. As a consequence, the distance between any other couple

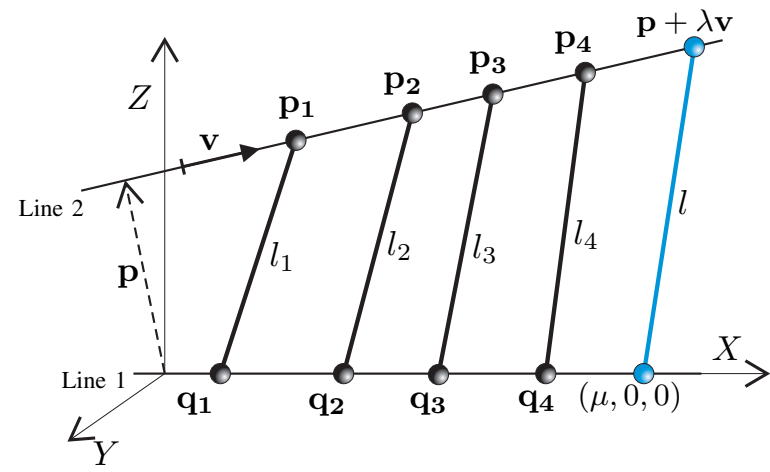

Fig. 3. Two lines are rigidly linked by fixing the distances between four couples of points on them. Hence, the distance between any other couple of points, one on each line, can be expressed as a function of these four distances. 
of points, one on each line, can be expressed in terms of these four distances. To realize this fact, take, without loss of generality, the $X$ axis of the reference frame coincident with one of the two lines, and the other line defined as $\mathbf{p}+\lambda \mathbf{v}$, where $\mathbf{p}=\left(p_{x}, p_{y}, p_{z}\right)$ is a point of the line and $\mathbf{v}=\left(v_{x}, v_{y}, v_{z}\right)$ is a unit vector along it. Then, the coordinates of the four couples of points can be expressed as $\mathbf{q}_{i}=\left(\mu_{i}, 0,0\right)$, and $\mathbf{p}_{i}=\mathbf{p}+\lambda_{i} \mathbf{v}$, and the squared distances between them as

$$
l_{i}^{2}=\left(p_{x}+\lambda_{i} v_{x}-\mu_{i}\right)^{2}+\left(p_{y}+\lambda_{i} v_{y}\right)^{2}+\left(p_{z}+\lambda_{i} v_{z}\right)^{2}
$$

for $i=1, \ldots, 4$.

Then, by subtracting $l_{i}^{2}, i=2,3,4$, from $l_{1}^{2}$, quadratic terms cancel yielding the linear system

$$
\begin{gathered}
\left(\begin{array}{ccc}
\lambda_{2} \mu_{2}-\lambda_{1} \mu_{1} & \mu_{2}-\mu_{1} & \lambda_{1}-\lambda_{2} \\
\lambda_{3} \mu_{3}-\lambda_{1} \mu_{1} & \mu_{3}-\mu_{1} & \lambda_{1}-\lambda_{3} \\
\lambda_{4} \mu_{4}-\lambda_{1} \mu_{1} & \mu_{4}-\mu_{1} & \lambda_{1}-\lambda_{4}
\end{array}\right)\left(\begin{array}{c}
p_{x} \\
v_{x} \\
w
\end{array}\right)= \\
\left(\begin{array}{c}
l_{1}^{2}-l_{2}^{2}-\mu_{1}^{2}+\mu_{2}^{2}-\lambda_{1}^{2}+\lambda_{2}^{2} \\
l_{1}^{2}-l_{3}^{2}-\mu_{1}^{2}+\mu_{3}^{2}-\lambda_{1}^{2}+\lambda_{3}^{2} \\
l_{1}^{2}-l_{4}^{2}-\mu_{1}^{2}+\mu_{4}^{2}-\lambda_{1}^{2}+\lambda_{4}^{2}
\end{array}\right),
\end{gathered}
$$

where $w=\mathbf{p} \cdot \mathbf{v}$. Then, there is a single solution for $p_{x}, v_{x}$, and $w$, if, and only if,

$$
\left|\begin{array}{ccc}
\lambda_{2} \mu_{2}-\lambda_{1} \mu_{1} & \mu_{2}-\mu_{1} & \lambda_{1}-\lambda_{2} \\
\lambda_{3} \mu_{3}-\lambda_{1} \mu_{1} & \mu_{3}-\mu_{1} & \lambda_{1}-\lambda_{3} \\
\lambda_{4} \mu_{4}-\lambda_{1} \mu_{1} & \mu_{4}-\mu_{1} & \lambda_{1}-\lambda_{4}
\end{array}\right| \neq 0 .
$$

The above condition can be rewritten as:

$$
\frac{\left(\lambda_{1}-\lambda_{3}\right)\left(\lambda_{2}-\lambda_{4}\right)}{\left(\lambda_{1}-\lambda_{4}\right)\left(\lambda_{2}-\lambda_{3}\right)} \neq \frac{\left(\mu_{1}-\mu_{3}\right)\left(\mu_{2}-\mu_{4}\right)}{\left(\mu_{1}-\mu_{4}\right)\left(\mu_{2}-\mu_{3}\right)} .
$$

In other words, the cross-ratios [3] of the four points on both lines must be different.

The squared distance between any other couple of points on both lines, say $\mathbf{q}=(\mu, 0,0)$ and $\mathbf{p}+\lambda \mathbf{v}$ (Figure 3), when subtracted from $l_{1}^{2}$, yields

$$
l^{2}=\lambda^{2}+\mu^{2}-2 \mu p_{x}-2 \lambda \mu v_{x}+2 \lambda w+l_{1}^{2}
$$

Then, the value for $l^{2}$ is unique if, and only if, (4) is satisfied. We can substitute any of the four distance constraints by another distance constraint between another couple of points on both lines so that they remain rigidly linked. Note that this substitution, which we call $L L$-transformation, defines a one-to-one transformation.

\section{Applying the LL-transformation to the basic 3-3 flagged design}

By repeating the $L L$-transformation four times, the basic 3-3 flagged parallel manipulator can be transformed into the 6-6 parallel manipulator shown in Figure 4.

Since the $L L$-transformation is one-to-one, the resulting 66 design should have the same number of forward kinematic solutions and the same kind of singularities than the basic 33 flagged manipulator. This is verified in the next section by showing that this assertion is true if, and only if, the resulting 6-6 design does not satisfy the cross-ratio condition.

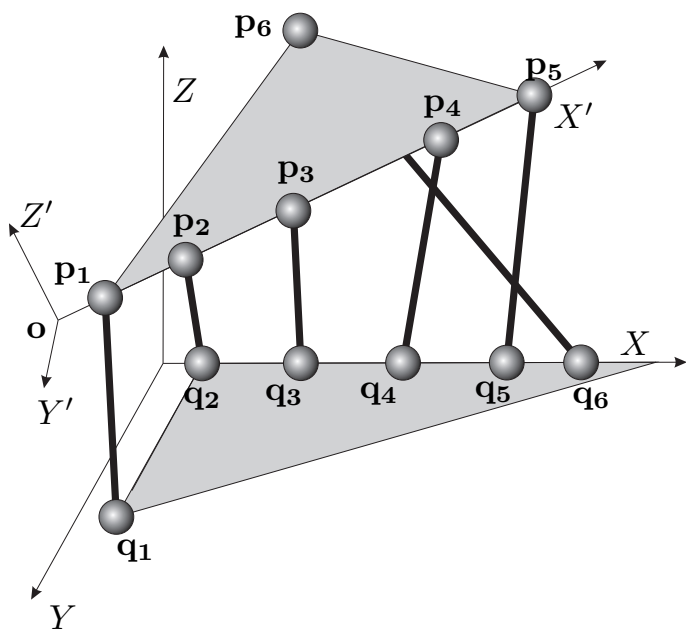

Fig. 4. The 6-6 design resulting from applying four times the $L L$ transformation to the 3-3 basic flagged manipulator.

\section{AnAlysis of THE OBTAINED 6-6 DESIGN}

\section{A. Forward kinematics}

The forward kinematics of the obtained 6-6 design was already solved in [16], but no singularity analysis was provided. As an alternative, we can solve the forward kinematics by applying the just introduced $L L$-transformation to pass from the 6-6 design to the 3-3 flagged manipulator, whose forward kinematics can be straightforwardly solved by a sequence of three trilateration as shown in Figure 5 (see [1] and [13] for details).
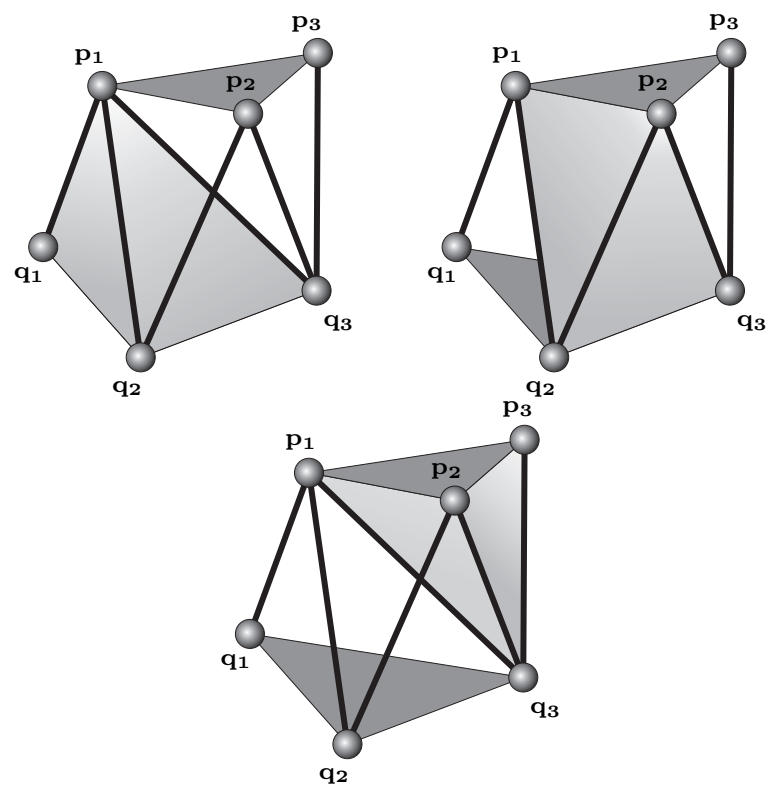

Fig. 5. The forward kinematics of the 3-3 flagged manipulator can be solved by a sequence of three trilaterations.

The first tetrahedron yields two positions for $\mathbf{p}_{1}$. The second trilateration gives other two solutions for point $\mathbf{p}_{2}$ and finally the last tetrahedron provides two more solutions for $\mathbf{p}_{3}$, so that the eight solutions for the position and orientation of the platform are determined. 


\section{B. Singularities}

The analysis of the singularities can be carried out by analyzing the determinant of the jacobian matrix whose columns are the Plücker coordinates of the leg lines [11].

The Plücker coordinates of a line can be expressed as $P l_{L}=(\mathbf{d}, \mathbf{d} \times \mathbf{p})$, where $\mathbf{p}$ is a vector pointing to a point on the line and $\mathbf{d}$ a unit vector along it (see [12], page 140).

According to the notation used in Figure 4, the platform mobile reference frame has its origin at $\mathbf{o}=\left(x_{o}, y_{o}, z_{o}\right)$ with respect to the base fixed reference frame, with orientation matrix $\mathbf{R}=(\mathbf{i}, \mathbf{j}, \mathbf{k})$. Local coordinates of platform points are $\mathbf{p}_{i}=\left(\lambda_{i}, 0,0\right)$ for $i=1 \ldots 5$, and $\mathbf{p}_{6}=\left(\lambda_{11}, \lambda_{12}, 0\right)$. Base points are $\mathbf{q}_{1}=\left(\mu_{11}, \mu_{12}, 0\right)$ and $\mathbf{q}_{i}=\left(\mu_{i}, 0,0\right)$ for $i=2 \ldots 6$.

Referring all points to the fixed reference frame $X, Y, Z$, the Plücker coordinates of the six leg lines are:

$$
\begin{aligned}
& P l_{L 1}=\left(\begin{array}{c}
i_{x} \lambda_{1}+x_{o}-\mu_{11} \\
i_{y} \lambda_{1}+y_{o}-\mu_{12} \\
i_{z} \lambda_{1}+z_{o} \\
-\left(i_{z} \lambda_{1}+z_{o}\right) \mu_{12} \\
\left(i_{z} \lambda_{1}+z_{o}\right) \mu_{11} \\
\left(i_{x} \lambda_{1}+x_{o}-\mu_{11}\right) \mu_{12}-\left(i_{y} \lambda_{1}+y_{o}-\mu_{12}\right) \mu_{11}
\end{array}\right) \\
& P l_{L 2}=\left(\begin{array}{c}
i_{x} \lambda_{2}+x_{o}-\mu_{2} \\
i_{y} \lambda_{2}+y_{o} \\
i_{z} \lambda_{2}+z_{o} \\
0 \\
\left(i_{z} \lambda_{2}+z_{o}\right) \mu_{2} \\
-\left(i_{y} \lambda_{2}+y_{o}\right) \mu_{2}
\end{array}\right), \quad P l_{L 3}=\left(\begin{array}{c}
i_{x} \lambda_{3}+x_{o}-\mu_{3} \\
i_{y} \lambda_{3}+y_{o} \\
i_{z} \lambda_{3}+z_{o} \\
0 \\
\left(i_{z} \lambda_{3}+z_{o}\right) \mu_{3} \\
-\left(i_{y} \lambda_{3}+y_{o}\right) \mu_{3}
\end{array}\right), \\
& P l_{L 4}=\left(\begin{array}{c}
i_{x} \lambda_{4}+x_{o}-\mu_{4} \\
i_{y} \lambda_{4}+y_{o} \\
i_{z} \lambda_{4}+z_{o} \\
0 \\
\left(i_{z} \lambda_{4}+z_{o}\right) \mu_{4} \\
-\left(i_{y} \lambda_{4}+y_{o}\right) \mu_{4}
\end{array}\right), \quad P l_{L 5}=\left(\begin{array}{c}
i_{x} \lambda_{5}+x_{o}-\mu_{5} \\
i_{y} \lambda_{5}+y_{o} \\
i_{z} \lambda_{5}+z_{o} \\
0 \\
\left(i_{z} \lambda_{5}+z_{o}\right) \mu_{5} \\
-\left(i_{y} \lambda_{5}+y_{o}\right) \mu_{5}
\end{array}\right) \text {, } \\
& P l_{L 6}=\left(\begin{array}{c}
i_{x} \lambda_{61}+j_{x} \lambda_{62}+x_{o}-\mu_{6} \\
i_{y} \lambda_{61}+j_{y} \lambda_{62}+y_{o} \\
i_{z} \lambda_{61}+j_{z} \lambda_{62}+z_{o} \\
0 \\
\left(i_{z} \lambda_{61}+j_{z} \lambda_{62}+z_{o}\right) \mu_{6} \\
-\left(i_{y} \lambda_{61}+j_{y} \lambda_{62}+y_{o}\right) \mu_{6}
\end{array}\right)
\end{aligned}
$$

Then, the outcome of factorizing the determinant of the jacobian is:

$$
\begin{gathered}
\operatorname{det}\left(J_{f}\right)=\lambda_{62} \mu_{12} \\
\left(x_{o} i_{y} j_{z}-x_{o} i_{z} j_{y}+\mu_{6} i_{z} j_{y}-\mu_{6} i_{y} j_{z}+\right. \\
\left.y_{o} i_{z} j_{x}-y_{o} i_{x} j_{z}+z_{o} i_{x} j_{y}-z_{o} i_{y} j_{x}\right) \\
\left(i_{y} z_{o}-y_{o} i_{z}\right) \\
\left(i_{z} \lambda_{1}+z_{o}\right) \\
\left(\left(\lambda_{2}-\lambda_{4}\right)\left(\lambda_{3}-\lambda_{5}\right)\left(\mu_{2}-\mu_{5}\right)\left(\mu_{3}-\mu_{4}\right)-\right. \\
\left.\left(\mu_{2}-\mu_{4}\right)\left(\mu_{3}-\mu_{5}\right)\left(\lambda_{2}-\lambda_{5}\right)\left(\lambda_{3}-\lambda_{4}\right)\right)
\end{gathered}
$$

Notice that, if $\lambda_{62}=0$, or $\mu_{12}=0$, the six joints on the platform, or on the base, are aligned. In both cases the platform becomes architecturally singular. Next, the remaining non-trivial factors are interpreted geometrically in terms of incidences between points, lines, and planes.

The first non-trivial factor is

$$
\begin{aligned}
J F_{1}= & x_{o}\left(i_{y} j_{z}-i_{z} j_{y}\right)-\mu_{6}\left(i_{y} j_{z}-i_{z} j_{y}\right)+ \\
& y_{o}\left(i_{z} j_{x}-i_{x} j_{z}\right)+z_{o}\left(i_{x} j_{y}-i_{y} j_{x}\right),
\end{aligned}
$$

which can be rewritten as

$$
J F_{1}=\left|\begin{array}{ccc}
i_{x} & j_{x} & x_{o}-\mu_{6} \\
i_{y} & j_{y} & y_{o} \\
i_{z} & j_{z} & z_{o}
\end{array}\right|,
$$

where $\left(i_{x}, i_{y}, i_{z}\right)$ and $\left(j_{x}, j_{y}, j_{z}\right)$ are two vectors spanning the platform plane, and $\left(x_{o}-\mu_{6}, y_{o}, z_{o}\right)$ is the vector $\left(\mathbf{o}-\mathbf{q}_{6}\right)$ in the base reference frame.

Conclusion 1 The first non-trivial factor in (6) is zero if, and only if, $\mathbf{q}_{6}$ lies on the platform plane.

Now, let $P l_{L}=(l, \bar{l})$ be the Plücker coordinates of line $L$. Then, given two lines, $G=(g, \bar{g})$ and $H=(h, \bar{h})$, the bilinear form $\Omega$ defined by

$$
\Omega(G, H)=g \cdot \bar{h}+\bar{g} \cdot h
$$

is zero if, and only if, $G$ and $H$ are coplanar (see [12], p. 140).

The Plücker coordinates of the two lines defining the $L L$ component are

$$
\begin{gathered}
P l_{B}=\left(\mathbf{q}_{\mathbf{2}}-\mathbf{q}_{\mathbf{4}},\left(\mathbf{q}_{\mathbf{2}}-\mathbf{q}_{\mathbf{4}}\right) \times \mathbf{q}_{\mathbf{2}}\right)=\left(\begin{array}{c}
\mu_{2}-\mu_{4} \\
0 \\
0 \\
0 \\
0 \\
0
\end{array}\right), \\
P l_{P}=\left(\mathbf{p}_{\mathbf{2}}-\mathbf{p}_{\mathbf{4}},\left(\mathbf{p}_{\mathbf{2}}-\mathbf{p}_{\mathbf{4}}\right) \times \mathbf{p}_{\mathbf{2}}\right)=\left(\begin{array}{c}
\left(\lambda_{2}-\lambda_{4}\right) i_{x} \\
\left(\lambda_{2}-\lambda_{4}\right) i_{y} \\
\left(\lambda_{2}-\lambda_{4}\right) i_{z} \\
\left(\lambda_{2}-\lambda_{4}\right)\left(i_{y} z_{o}-i_{z} y_{o}\right) \\
\left(\lambda_{2}-\lambda_{4}\right)\left(i_{z} x_{o}-i_{x} z_{o}\right) \\
\left(\lambda_{2}-\lambda_{4}\right)\left(i_{x} y_{o}-i_{y} x_{o}\right)
\end{array}\right)
\end{gathered}
$$

Then, substituting them in (9), we obtain

$$
\Omega\left(P l_{B}, P l_{P}\right)=\left(\mu_{2}-\mu_{4}\right)\left(\lambda_{2}-\lambda_{4}\right)\left(i_{y} z_{o}-y_{o} i_{z}\right),
$$

which is zero if, and only if, the second non-trivial factor in (6),

$$
J F_{2}=i_{y} z_{o}-y_{o} i_{z},
$$

is zero. Notice that if $\mu_{2}-\mu_{4}=0$ or $\lambda_{2}-\lambda_{4}=0$ the lines of the $L L$-component are not well-defined so different points must be chosen to define $P l_{B}$ or $P l_{P}$.

Conclusion 2 The second non-trivial factor in (6) is zero if, and only if, the LL component is planar.

The third non-trivial factor in (6) is

$$
J F_{3}=i_{z} \lambda_{1}+z_{o},
$$

which clearly corresponds to the $z$ coordinate of point $\mathbf{p}_{\mathbf{1}}$ in the base reference frame. 
Conclusion 3 The third non-trivial factor in (6) is zero if, and only if, $\mathbf{p}_{1}$ lies on the base plane

The last factor is

$$
\begin{aligned}
J F_{4}= & \left(\lambda_{2}-\lambda_{4}\right)\left(\lambda_{3}-\lambda_{5}\right)\left(\mu_{2}-\mu_{5}\right)\left(\mu_{3}-\mu_{4}\right)- \\
& \left(\mu_{2}-\mu_{4}\right)\left(\mu_{3}-\mu_{5}\right)\left(\lambda_{2}-\lambda_{5}\right)\left(\lambda_{3}-\lambda_{4}\right)
\end{aligned}
$$

which is exactly the expression of the cross-ratio relation in (4), for points $\left(\mathbf{p}_{2}, \mathbf{p}_{3}, \mathbf{p}_{4}, \mathbf{p}_{5}\right)$ and $\left(\mathbf{q}_{2}, \mathbf{q}_{3}, \mathbf{q}_{4}, \mathbf{q}_{5}\right)$.

Conclusion 4 The fourth factor is zero if, and only if, the cross-ratio relation between the joints in the LL component is satisfied.

Recalling the definition of flagged manipulator at the beginning of Section II, we can say that the obtained 66 design is a flagged manipulator, the only difference with respect to all other flagged parallel manipulators presented to date being the possibility that it becomes architecturally singular when the cross-ratio condition is satisfied.

\section{DERIVING MORE FlaGged MANiPUlators}

\section{A. The two transformations applied}

In the previous work [1], the family of flagged designs which are not architecturally singular for any parameter values was derived. This was attained by repeatedly applying the transformation shown in Figure 6 to the basic 3-3 flagged manipulator. Such transformation can be applied to any pair of legs sharing an endpoint, and it consists in moving one of the nonshared endpoints along the line passing through the other nonshared endpoint (see [1] for details). The interest of this transformation is that it leaves invariant the relative position between a point and a line, and, therefore, preserves the forward kinematics of any parallel platform containing a $P L$ (Point-Line) component (Section II-A).

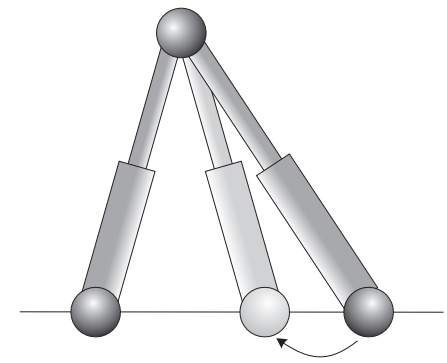

Fig. 6. PL-transformation preserving the kinematics of a $P L$ component.

Similarly, the LL-transformation presented in Section IIB leaves invariant the relative position between two lines and, therefore, preserves the forward kinematics of any parallel platform containing a $L L$ (Line-Line) component. The important difference between both transformations is that, whereas the former doesn't introduce any architecture singularity -except for the trivial overlapping of the two legs-, the latter may introduce the "equal cross ratios" singularity.

Let us study the possible topologically-distinct outcomes of this second transformation. Table I shows the eight possible arrangements of four legs with their endpoints aligned both in the base and the platform. Of them, five cases are never architecturally singular (i.e., the cross ratios of points in the base and the platform are necessarily different), two more cases are always singular, and only one case that with no shared endpoints between the legs - may be architecturally singular (when the cross ratios of points in the base and the platform are equal). This can be easily proved by realizing that the cross ratio of arrangements with coincident points is 0,1 or $\infty$ depending on the cases, while the cross ratio of four distinct points never takes these values.

TABLE I

POSSIBLE OUTCOMES OF THE $L L$-TRANSFORMATION

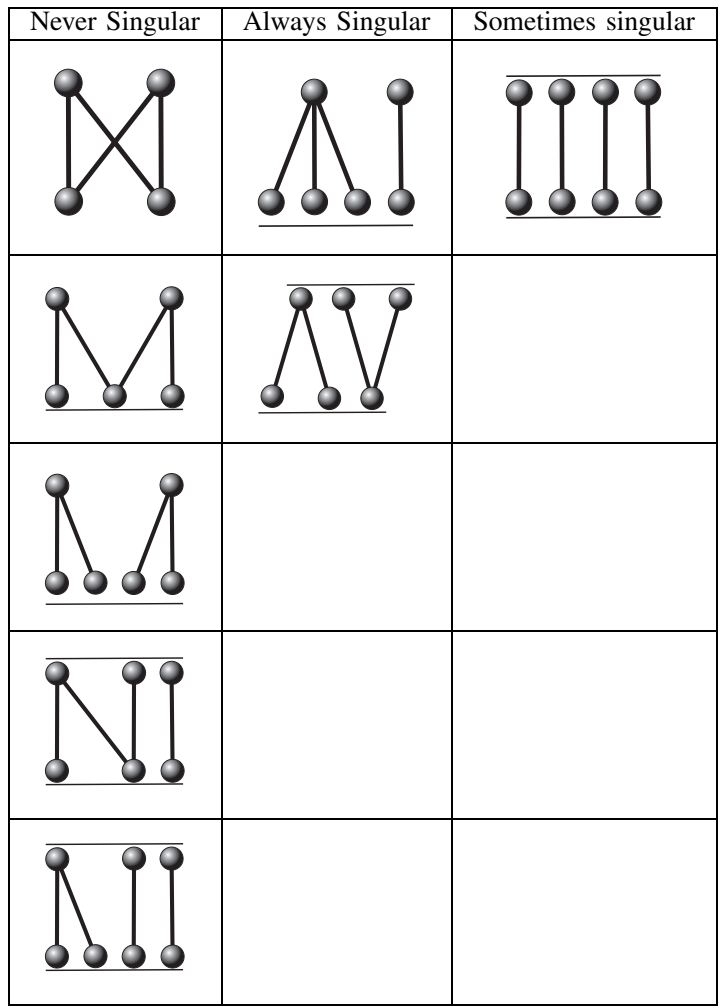

\section{B. The enlarged family of flagged manipulators}

By applying the two transformations above, the extended family of possibly architecturally-singular flagged manipulators is derived from the 6-6 flagged manipulator (refer to Table II). Let us describe how the family is generated. Starting from the five "never singular" and the one "sometimes singular" $L L$-components in Table I, two additional legs are added to every component designs in all possible manners, so that each leg has an endpoint in either the upper or lower line, and the other endpoint in general position. From the upmost "never singular" design in Table I, 3 topologically different manipulators are obtained. Likewise, the remaining four "never singular" designs give rise to $7,5,10$ and 10 different manipulators, respectively. These 35 flagged manipulators are free of architecture singularities. Now, from the "sometimes singular" design in Table I, 4 additional flagged manipulators are obtained, which are prone to the "equal 
TABLE II

EXPANDED FAMILY OF FLAGGED PARALLEL MANIPULATORS

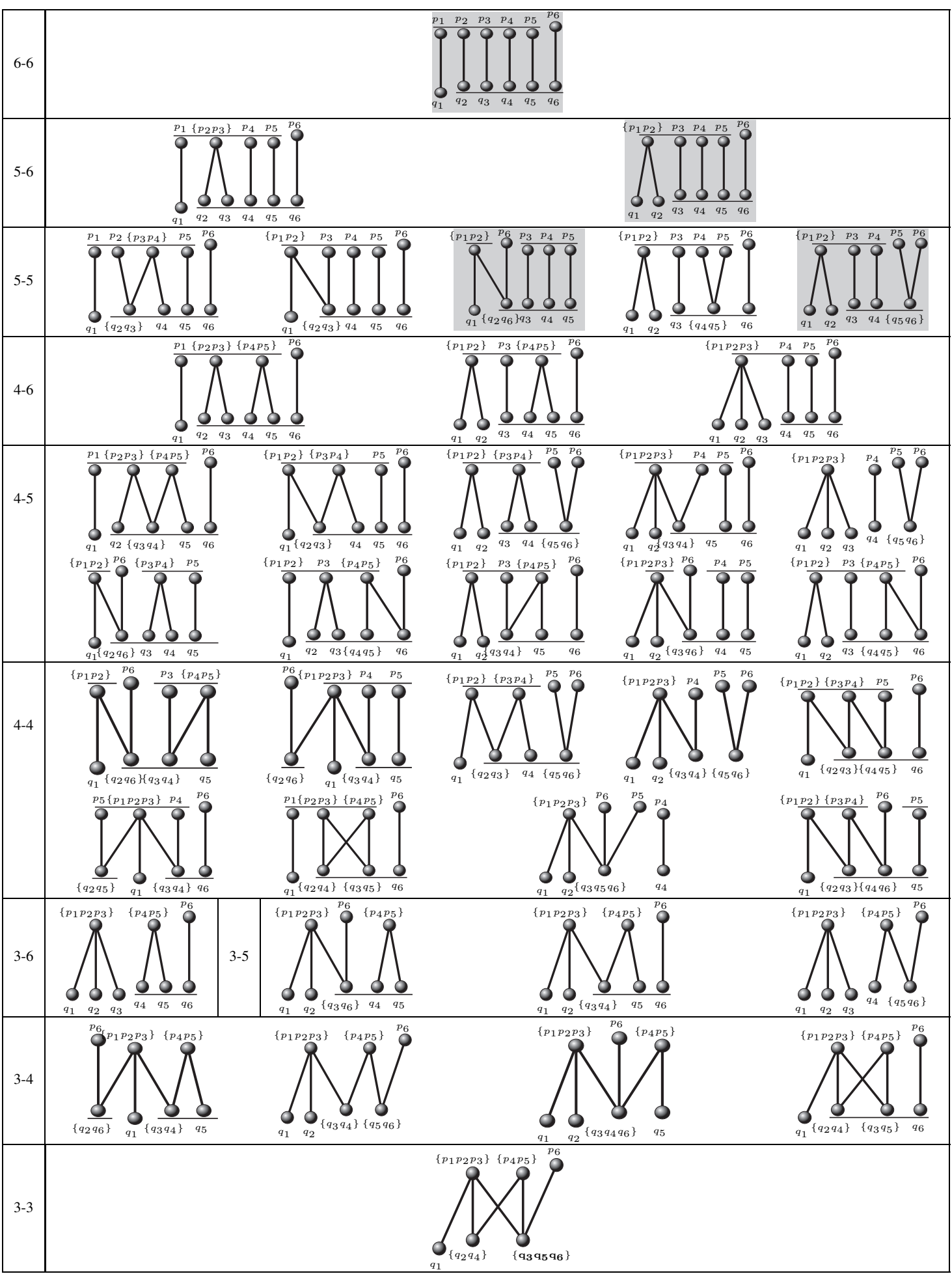


cross ratios" singularity, and, thus, become architecturally singular for some specific conjunction of parameter values.

The 39 manipulators thus obtained have four endpoints aligned both in the base and the platform, and they can be thought of as resulting from applying the $L L$-transformation to the 6-6 flagged manipulator. Then, by applying the $P L$ transformation, some of these manipulator designs can be made more general by misaligning some of their endpoints. The resulting manipulators conform the enlarged family of flagged parallel manipulators displayed in Table II.

The interesting point is that the previously-known set of flagged manipulators [1] has been enlarged with the 4 new manipulator designs stemming from the "sometimes singular" $L L$-component, highlighted in grey in Table II, which incidentally are among the less specialized ones (i.e., they have fewest endpoint merges).

Note that every manipulator in Table II has its endpoints labelled so that the analysis carried out for the 6-6 design in Section III-B becomes applicable to them all. In fact, the factorization of the Jacobian for all instances contains the factors $J F_{1}, J F_{2}$ and $J F_{3}$ in Section III-B in exactly the same form. The architecture singularity factor $J F_{4}$ appears only for the four manipulators highlighted in grey, and its expression varies depending on the design. This is due to the disalignments introduced by the $P L$-transformation. For instance, the cross ratio in the lower line of the 2nd 5-6 design involves, as fourth point, that at the intersection of the base line with the line of the $P L$-component. Similarly, for the 5th 5-5 design, both cross ratios involve intersections with $P L$-component lines.

It is worth noting that the endpoints that do not appear aligned in Table II are assumed to be in general position. If they were not, some other architecture singularities could either appear or disappear. For example, the alignment of the three endpoints of a tripod leads always to an architecture singularity. On the contrary, the alignment of endpoints $\mathbf{q}_{1}$, $\mathbf{q}_{2}$ and $\mathbf{q}_{3}$ in the 2nd 5-6 design removes the architecture singularity. Coherently, this design and the 5th 5-5 one appeared with additional alignments in the set of architecturesingularity-free flagged manipulators derived in [1].

\section{CONCLUSIONS AND FURTHER RESEARCH}

In this paper, the nice properties of flagged manipulators have been extended to four additional designs, namely, one 6-6, one 5-6 and two 5-5 manipulators. Moreover, it has been shown that the family of such manipulators is now complete, in the sense that no other Stewart-Gough parallel platforms can have singularity loci with the well-behaved structure of flagged manipulators. This nice structure allows a decomposition of the singularity loci with a unique topology irrespective of the metrics of each particular design. The family completeness follows from an analysis of the metric architecture singularities that may arise for flagged designs, the only one being the "equal cross ratios" singularity (refer to the Introduction). Thus, the only kinematic transformation that could enlarge the family at the expense of introducing designs prone to this architecture singularity is the $L L$ trasformation considered in this work.

As for the envisaged future work, we are studying other families of parallel manipulators having singularity spaces with a topology as close as possible to that of flagged manipulators. This is addressed from both geometric and algebraic viewpoints, the latter entailing finding invariant factorizations of the jacobian determinant.

\section{ACKNOWLEDGMENTS}

The authors gratefully acknowledge the contribution of Maria Alberich-Carramiñana and Josep M. Porta for fruitful discussions about this work.

\section{REFERENCES}

[1] M. Alberich-Carramiñana, F. Thomas, and C. Torras, "Flagged Parallel Manipulators," IEEE Trans. on Robotics, Vol. 23, No. 5, 2007, pp. 1013 1023.

[2] M. Alberich-Carramiñana, F. Thomas, and C. Torras, "On Redundant Flagged Manipulators," Proc. IEEE Intl. Conf. on Robotics and Automation, 2006, pp. 783-789.

[3] R. Courant and H. Robbins, What is Mathematics?, (Section IV.3), Oxford Univesity Press, 1996.

[4] B. Dasgupta and T.S. Mruthyunjaya, "The Stewart Platform Manipulator: a Review," Mechanism and Machine Theory, Vol. 35, 2000, pp $15-40$.

[5] M.L. Husty and A. Karger, "Self-Motions of Griffis-Duffy Type Parallel Manipulators," Proc. IEEE Intl. Conf. on Robotics and Automation, 2000, pp. 7-12.

[6] M.L. Husty and A. Karger, "Architecture Singular Parallel Manipulators and Their Self-Motions," in Advances in Robot Kinematics, J. Lenarcic and M. M. Stanisic (eds.), Kluwer Academic Publishers, 2000, pp. 355364.

[7] A. Karger, "Architecture Singular Planar Parallel Manipulators," Mechanism and Machine Theory, Vol. 38, 2003, pp. 1149-1164.

[8] X. Kong and C.M. Gosselin, "Classification of 6-SPS Parallel Manipulators According to Their Components," Proc. ASME Des. Eng. Tech. Conf., 2000.

[9] H. Li, C.M. Gosselin, M.J. Richard, and B.M. St-Onge, "Analytic Form of the Six-Dimensional Singularity Locus of the General Gough-Stewart Platform," Journal of Mechanical Design, Vol. 128, No. 1, 2006, pp. 279-287.

[10] O. Ma and J. Angeles, "Architecture Singularities of Platform Manipulators," Proc. IEEE Intl. Conf. on Robotics and Automation, 1991.

[11] J.-P. Merlet, Parallel Robots, Springer, 2000.

[12] H. Pottmann and J. Wallner, Computational Line Geometry Mathematics + Visualization, Chap. 2, Springer, 2001, pp. 133-141.

[13] F. Thomas, E. Ottaviano, L. Ros, and M. Ceccarelli, "Performance Analysis of a 3-2-1 Pose Estimation Device," IEEE Trans. on Robotics, Vol. 21, No. 3, 2005, pp. 288-297.

[14] C. Torras, F. Thomas, and M. Alberich-Carramiñana, "Stratifying the Singularity Loci of a Class of Parallel Manipulators," IEEE Trans. on Robotics, Vol. 22, No. 1, 2006, pp. 23-32.

[15] A. Wolf and M. Shohan, "Investigation of Parallel Manipulators Using Linear Complex Approximation,” Journal of Mechanical Design. Trans. of the ASME, Vol. 125, 2003, pp. 564-572.

[16] C. Zhang and S.M. Song, "Forward Kinematics of a Class of Parallel (Stewart) Platforms with Closed-Form Solutions," Proc. IEEE Intl. Conf. on Robotics and Automation, 1991, pp. 2676-2681. 